\title{
A Noncommunicative Memory-Pushing Fuzzy Control Strategy for Sensorless Multirobot Systems
}

\author{
Lin Zhang $\mathbb{D},{ }^{1,2}$ Xianhua Zheng $\mathbb{D},{ }^{1}$ Shang Feng, ${ }^{3}$ and Lingling Su $\mathbb{D}^{4}$ \\ ${ }^{1}$ School of Mechanical and Electrical Engineering, Yangtze Normal University, Chongqing 408100, China \\ ${ }^{2}$ Jiangsu Key Laboratory of Special Robot Technology, Changzhou 213022, China \\ ${ }^{3}$ School of Computer Science and Technology, Harbin Institute of Technology, Harbin 150001, China \\ ${ }^{4}$ College of Science, North China University of Technology, Beijing 100144, China \\ Correspondence should be addressed to Lin Zhang; lin.zhang_2014@hotmail.com and Lingling Su; sll506@bit.edu.cn
}

Received 28 December 2019; Accepted 19 June 2020; Published 24 August 2020

Academic Editor: Xianming Zhang

Copyright (C) 2020 Lin Zhang et al. This is an open access article distributed under the Creative Commons Attribution License, which permits unrestricted use, distribution, and reproduction in any medium, provided the original work is properly cited.

\begin{abstract}
Considering the characteristics of equipment on underground fully mechanized coal mining face, a multirobot system, which takes heavy-duty mobile support robot (HMSR) as the pushing robot and middle trough (MT) as the manipulated object, is established. To overcome the problem of unstable communication and potential pressure loss, a memory-pushing fuzzy control strategy is proposed to achieve better practical performance without human-guided operations. The pushing dynamics without communication is derived to proof the convergence of the dynamic system, and the time-based memory-pushing fuzzy model is built for compensating the potential pressure loss. Finally, the proposed control strategy is simulated in virtual environment, which integrates our pushing dynamics, and an industrial experiment is demonstrated as well. Both the simulation and industrial experiments show the efficiency and feasibility of the proposed method.
\end{abstract}

\section{Introduction}

As one of the most intelligent and important equipment on underground fully mechanized coal mining face, hydraulic support can be abstracted to heavy-duty mobile support robot (HMSR) due to its loadable characteristics as well as mobility through pushing and advancing. As shown in Figure 1(a), the layout of equipment on underground fully mechanized coal mining face is presented. There are three types of equipment: mining machine, scraper conveyer, and hundreds of HMSRs. Mining machine is the main equipment for excavation, while scraper conveyer and HMSRs are assistance equipment for continuous mining. Scraper conveyor is composed of many middle troughs (MTs) connected to each other. At each working cycle, firstly, HMSR will cooperatively push corresponding MT forward. Then, HMSRs advance themselves forward to align with neighbors. Since the pushing-advancing process is completed by hundreds of HMSRs and corresponding number of MTs, the system is essentially a multirobot system, and the process described above is much like planar pushing manipulation. Our purpose of this article is to study the pushing manipulation approach of multirobot system, which takes HMSR as the pushing robot and MT as the manipulated object. The physical model of the multirobot system is shown in Figure 1(b). It is notable that manipulated objects are physically connected with neighbors, which dramatically increases the complexity of the problem. Additionally, due to unstable communication, terrible working environment, different geological conditions, and pressure loss of hydraulic system, the pushing result is always out of alignment. Thus, the problem remains a great challenge although a lot of valuable research on cooperative manipulation has been conducted.

In recent years, research on multirobot systems has attracted much attention [1], which is widely applied in robotics [2], optimization [3], collective cell biology [4], etc. The latest impressive research on multirobot systems, which is inspired by biological organisms, demonstrates particle robotics by incorporating many loosely coupled 

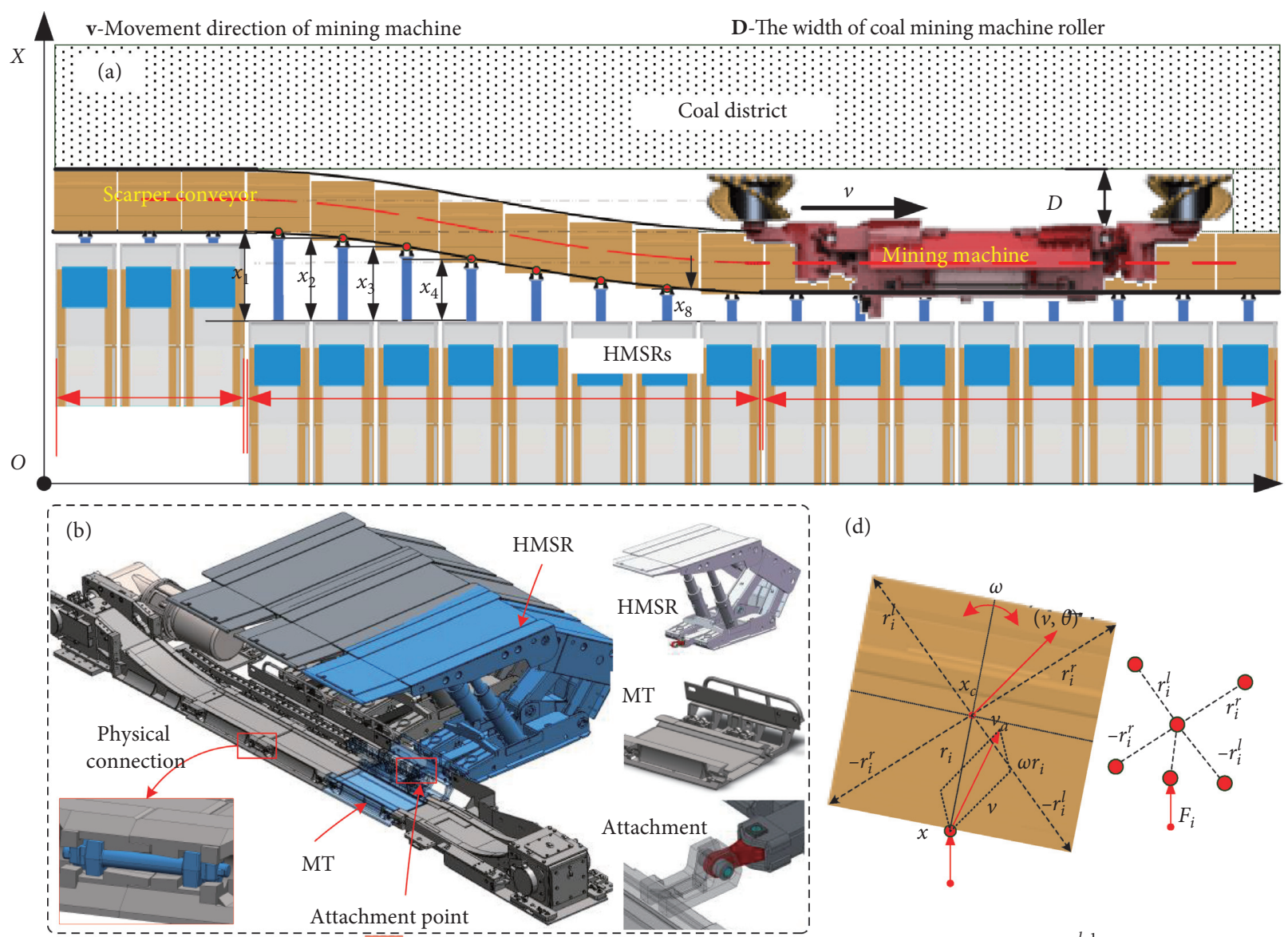

(d)

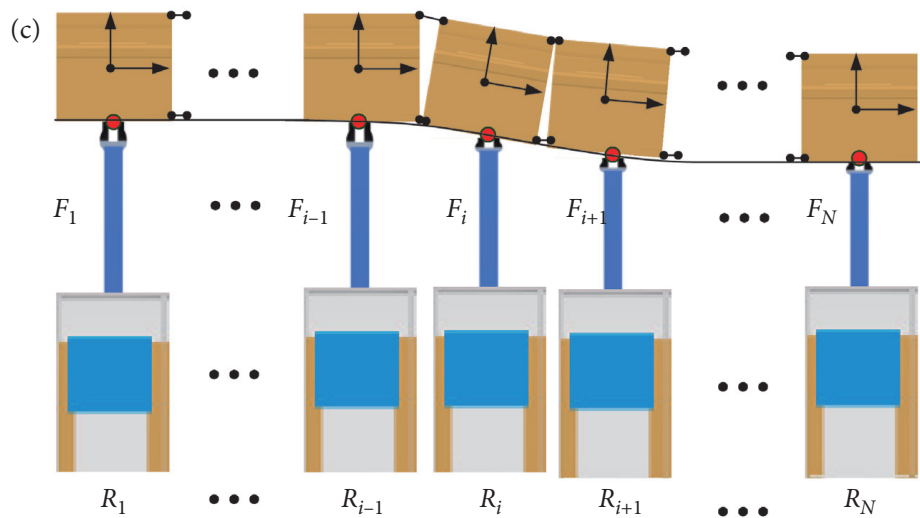

(e)
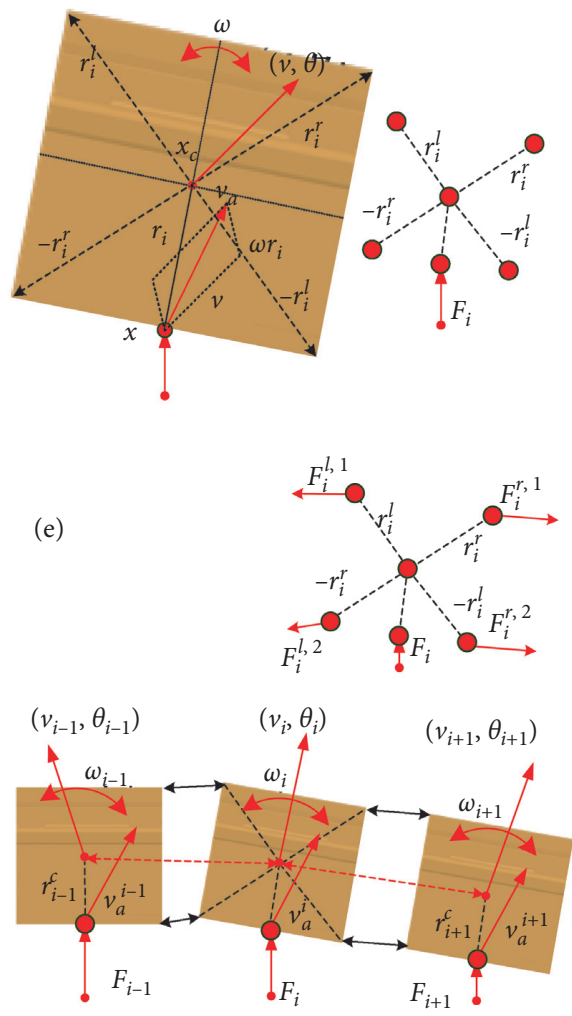

Figure 1: Description of the multirobot system on underground fully mechanized coal mining face; (a) layout of equipment on the underground fully mechanized coal mining face; scraper conveyor is composed of many middle troughs (MTs) connected to neighbors. HMSRs push corresponding MT forward or backward; (b) physical model of the multirobot system, MTs are physically connected with neighbors; (c) planar pushing manipulation model of the multirobot system, the pushing objective is to push all MTs to align with neighbors; (d) mechanics of single MT without physical connection; (e) mechanics of multiple MTs with physical connections, each MT is connected with two neighbors.

components. The individual component in the system moves stochastically without direction control. However, the system is capable of robust locomotion and object transportation after cooperative control [5]. The stochastic mechanism in nature provides extraordinary approach for cooperative control; e.g., the cooperative behavior in transportation of ant group is one of the most adopted mechanisms for cooperative control. McCreery and Breed [6] studied the behavioral mechanisms that lead to cooperation in the ant group. This research discovers four 
phases of cooperative transport and proposes two measures of cooperative transport efficiency. Gelblum et al. [7] researched how ant group optimize collective transport, and the results show that efficient group-level processes can arise from transient amplification of individual-based knowledge. Thus, it is important for carriers to conform to their efforts and align their forces. Similarly, Feinerman revisited this problem, and a more detailed research on ants' cooperative transportation is published in Nature [8]. Feinerman also discovers that cooperative transport of furniture requires some nontrivial communication, and recent advances have drawn attention on this communication already. In this work, a coupled-carriers model based on Ising model is proposed for statistical physics of interacting particles with cognitive capabilities of individuals. From the above outstanding literature studies, we found that the key in multirobot systems is cooperation between individuals, and the communication between individuals also affects the cooperative performance. Wang and Schwager $[9,10]$ proposed a method for multirobot manipulation with only local measurements, which means that the communication between individuals is not required. In 2016, Wang applied this method in cooperative pushing manipulation of N-robot transport system (ANTS) without communication [11]. The analysis and simulation of two ANTS implementations prove the state-of-the-art method. Considering sensorless characteristic and unstable communication condition, these works inspired us that noncommunicative cooperative control is essential for pushing manipulation of HMSR.

The problem of pushing manipulation has been researched in 1999 by Rezzoug and Gorce [12], and massive documents have recorded achievements on its model establishment and controller design $[13,14]$. For example, Ma and Rodriguez [15] built an anisotropic friction model to better understand the variability and predictability of planar friction, and experiments were conducted for comparison with more standard isotropic friction models. Aimed at controlling pushing manipulation, a task-adaptive model and corresponding predictive controller are proposed for different accuracy requirements of task [16]. Considering the problem of multirobot transport system, a reinforcement learning based method is proposed for box-pushing tasks, which is enhanced by adaptive state aggregation Q-Learning method [17], and performance comparisons between reinforcement learning algorithms of multirobot pushing task are analyzed by Rahimi et al. [18] as well. However, these researches are focused on single object pushing manipulation. The manipulation problem of multiple objects by pushing is only studied in a few papers till now. Harada et al. are one of these frontiers. In their paper, an edge model was proposed to push a chain of $N$ objects stably [19]. Both sliding and rotation motions are considered in the final experiments, and the result proves the effectiveness of proposed method. Aimed at service robot, human body is simplified as an object with two-link model, and the kinematics of the free-ended two-rigid-links are analyzed in Japan [20]. Since existing affordance models cannot cope with multiple objects that may interact during action execution, a relational affordance model is proposed for multiple-object manipulation, and the proposed model possesses four advantages compared with traditional model [21]. Additionally, multiple objects with interlink connections are also reviewed. Manipulation planning of multiple interlinked deformable linear objects provides potential application in aerospace and automotive assembly [22]. As a specified application, i.e. aircraft assembly, mathematical formulation for attaching interlinked deformable linear objects to clamping points is proposed, and this formulation can be used for shape computation and manipulation planning [23].

As to control method of pushing manipulation in complex working environment, abundant and valuable researches have been published. Unfortunately, mathematic control model may be nonlinear or unavailable in most practical cases. At this time, fuzzy control method is appropriate to solve the problem [24-26]. With the help of Takagi-Sugeno fuzzy model, a new robust fuzzy schedulerbased fault tolerant controller is proposed to tackle multivariable nonlinear systems [27]. Aimed at earthquake resilient structures, a fuzzy logic controller, which continuously varies damping characteristics, is developed to control transient vibrations of semiactive tuned mass damper (TMD) [28]. For large-scale nonlinear systems, the detailed mathematic model is extremely difficult to obtain, because of unknown nonlinear functions, unknown deadzones, and unmeasurable state variables. Thus, a robust adaptive fuzzy decentralized output feedback control approach is proposed, and the effectiveness of the proposed controller is illustrated through simulation example [29]. In industrial applications, the adaptability of fuzzy controller is much needed. A robust adaptive control method for nonlinear systems subject to uncertainties is proposed based on an adaptive dynamic surface control [30], and a neural network enabled adaptive control system is proposed to improve the steel-casting performance based on fuzzy control method [31]. Concerning trajectory tracking problem, a backstepping-based adaptive controller is proposed for nonaffine stochastic nonlinear switched system [32]. Towards pure-feedback large-scale nonlinear systems, the communication between each control unit limits the control performance. Then, the finite-time event-triggered $H_{\infty}$ control problem for T-S Markov jump fuzzy system is studied to reduce communication burden [33]. This can also be solved by decentralized control. Thus, an adaptive fuzzy decentralized controller is constructed based on the investigation of adaptive fuzzy decentralized control [34]. Aimed at designing $H_{\infty}$ filters for standard T-S fuzzy systems, Cheng and Yang [35] propose a Nonfragile $H_{\infty}$ filter designing method with multiplicative gain variations. Since T-S fuzzy model can be employed to represent nonlinear system in both the continuous-time and discrete-time contexts, the problem of robust dynamic output feedback control for discrete-time nonlinear systems with parametric uncertainties is studied [36]. The proposed method is capable of effectively handling the parametric uncertainties and fuzzy system with immeasurable premise variables. Inspired by this control strategy, cooperative pushing 
manipulation of HMSRs can be reformalized from a totally distributed and autonomous control structure ignoring communication. Therefore, fuzzy logic control strategy can provide an essential solution for such problem.

Based on reviews of the above valuable research studies, it can be concluded that pushing manipulation of sensorless HMSRs without communication remains a great challenge. On the one hand, current researches on pushing manipulation are focused on single manipulated object. However, the manipulated object in this paper is multiple physical connected rigid bodies, and the complexity of pushing manipulation is dramatically increased due to coupling effect between neighboring MTs. On the other hand, the sensorless characteristics of some HMSRs and harsh working environment with unstable communication make it impossible to construct a close-loop control architecture. Thus, further research is essentially necessary to provide potential cooperative pushing approach for totally distributed multirobot systems.

Bearing the above observations in mind, we propose a memory-pushing fuzzy control strategy for sensorless HMSRs. Theoretically, the proposed strategy remains available even though the communication is totally disabled. The pushing dynamics is derived, and the convergence of pushing dynamics is proved considering noncommunicative situation. After comprehensive trade-off of cost and safety, a virtual environment of underground fully mechanized coal mining face is established based on pushing dynamics. Then, the pushing time-based memory-pushing fuzzy controller is developed for noncommunicative pushing manipulation, and the implemented controller is tested in virtual environment. Finally, the industrial experiment is demonstrated with one cutting round.

There are two highlights of this paper: (1) The noncommunicative pushing dynamics is derived for sensorless multirobot system, i.e., HMSRs, which manipulate multiple physical connected objects, i.e., MTs. (2) The timebased memory-pushing fuzzy control strategy is proposed for HMSR, which compensates the drawbacks of sensorless characteristics and pressure loss. The rest of the article is organized as follows. In Section 2, the pushing dynamics without communication is presented. In Section 3, the details about memory-pushing fuzzy control strategy, namely, the memory-pushing model, fuzzy controller, and its implementation, are described. A prototype system integrates pushing dynamics, and an example is demonstrated in Section 4. Finally, conclusions are summarized in Section 5.

\section{Pushing Dynamics without Communication}

Suppose that pushing dynamics is considered within a planar region $Q \in \mathbb{R}^{2}$, as shown in Figure $1(\mathrm{c})$. The schematic diagram of pushing dynamics is presented. The mass of $i$ th MT is denoted as $M_{i}$ and moment of inertia is $J_{i}$. The pose of each MT can be described as two parts: position of mass center $x_{c} \in \mathbb{R}^{2}$ and orientation $\theta \in \mathbb{R}^{1}$.
Generally, three kinds of friction should be considered, static friction, viscous friction, and kinetic friction, and coefficients are represented as $u_{s}, u_{v}$ and $u_{k}$ respectively. In our case, MTs are directly pushed on ground; thus, both static friction and kinetic friction are considered. The acceleration of gravity is $g$. Our problem is that $N$ HMSRs, which are denoted as $R_{i}, i \epsilon\{1,2, \ldots, N\}$, try to transport multiple physical connected MTs by pushing in Q. Each HMSR is capable of (1) pushing the corresponding MT to the next destination according to neighboring pose; (2) sensing the velocity and acceleration $\left(v=\dot{x}_{c}\right.$ and $\left.\dot{v}\right)$ of the corresponding MT with Inertial Measurement Unit (IMU); (3) measuring the pushing force $F_{i}$ and torque $T_{i}$ with local measurement. However, all the robots cannot communicate with each other, and only the first moving HMSR knows the next destination of pushing manipulation. The first moving HMSR is not fixed, and it can be specified by a program or guided by a human either. The objective is taking the first moving HMSR's force as input and generating a sequence of forces corresponding to each HMSR aligning in the same direction. In order to evaluate the alignment of all the pushed MTs, two criterions should be considered: (1) all pushed MTs should keep orientation the same; (2) all HMSRs should keep zero-torque at the attachment point with corresponding MT. Based on these considerations, pushing dynamics can be detailed as follows.

2.1. Individual MT Dynamics. For individual MT, as shown in Figure 1(d), the movement can be described as translational velocity $v$ and angular velocity $\omega$ under the forces from HMSRs and the environment. According to Newton's second law, translational dynamics can be written as follows:

$$
M_{i} v_{i}=F_{i}-\mu_{k} M_{i} g \frac{v}{\|v\|} .
$$

It can be discrete approximation by Euler's method:

$$
M_{i} \frac{v_{i}^{t+1}-v_{i}^{t}}{\Delta t}=F_{i}-\mu_{k} M_{i} g \frac{v_{t}}{\left\|v_{t}\right\|} .
$$

Since MT has geometric extension around mass center, two types of torque are studied to characterize rotational dynamics: one is associated with friction, and the other is associated with HMSRs' force. The frictional torque is derived via calculus method. Firstly, the static friction is neglected, then velocity on an arbitrary point of MT can be written as $v_{a}=v+\omega \times r_{i}$, where $v_{a}$ is the absolute velocity, $v$ is the translational velocity at mass center $x_{c}$, and $r_{i}$ is the vector pointing from $x_{c}$ to current point $x$. If density of MT is denoted as $\rho$, then kinetic frictional force can be calculated as follows:

$$
F_{v}=-\mu_{k} M_{i} g \frac{v_{a}}{\left\|v_{a}\right\|},
$$

And the kinetic frictional torque can be derived as 


$$
\begin{aligned}
T_{f} & =-\int_{S} \mu_{k} \rho g r_{i} \frac{v_{a}}{\left\|v_{a}\right\|} \mathrm{d} x=-\int_{S} \mu_{k} \rho g r_{i} \frac{v+\omega \times r_{i}}{\left\|v_{a}\right\|} \mathrm{d} x \\
& =-\mu_{k} g \int_{S} \rho\left(x-x_{c}\right) \frac{v}{\left\|v_{a}\right\|} \mathrm{d} x-\mu_{k} g \int_{S} \rho \frac{\omega \times r_{i}^{2}}{\left\|v_{a}\right\|} \mathrm{d} r \\
& =-\frac{\mu_{k} g}{\left\|v_{a}\right\|} \int_{S} \rho x \times v \mathrm{~d} x+\frac{\mu_{k} g x_{c}}{\left\|v_{a}\right\|} \times v \int_{S} \rho \mathrm{d} x-\mu_{k} g \int_{S} \rho \frac{\omega \times r_{i}^{2}}{\left\|v_{a}\right\|} \mathrm{d} r \\
& =-\frac{\mu_{k} g}{\left\|v_{a}\right\|}\left(\int_{S} \rho x \mathrm{~d} x\right) \times v+\frac{\mu_{k} g x_{c}}{\left\|v_{a}\right\|} \times v \int_{S} \rho \mathrm{d} x-\mu_{k} g \int_{S} \rho \frac{\omega \times r_{i}^{2}}{\left\|v_{a}\right\|} \mathrm{d} r \\
& =-\frac{\mu_{k} g}{\left\|v_{a}\right\|}\left(\int_{S} \rho x \mathrm{~d} x\right) \times v+\frac{\mu_{k} M g x_{c}}{\left\|v_{a}\right\|} \times v-\left(\frac{\mu_{k} g}{\left\|v_{a}\right\|} \int_{S} \rho r_{i}^{2} \mathrm{~d} r\right) \omega \\
& =-\frac{\mu_{k} g}{\left\|v_{a}\right\|} M x_{c} \times v+\frac{\mu_{k} M g x_{c}}{\left\|v_{a}\right\|} \times v-\left(\frac{\mu_{k} g}{\left\|v_{a}\right\|} \int_{S} \rho r_{i}^{2} \mathrm{~d} r\right) \omega .
\end{aligned}
$$

Finally, we obtain the frictional torque. $T_{f}=-\left(\left(\mu_{k} g\right)\right.$ $\left.\left.\left\|v_{a}\right\|\right) \int_{S} \rho r_{i}^{2} \mathrm{~d} r\right) \omega=-\left(\mu_{k} g /\left\|v_{a}\right\|\right) J_{i} \omega$.

The HMSR's torque is much easier in this case, because each object is centrosymmetric around mass center $x_{c}$. If HMSR's force is not pointing to $x_{c}$, then HMSR's torque is non-zero. Thus, the overall rotational dynamics can be written as follows:

$$
J_{i} \dot{\omega}=T_{i}-T_{f}=F_{i} \times r_{i}-\frac{\mu_{k} g}{\left\|v_{a}\right\|} J_{i} \omega
$$

2.2. Multiple MTs Dynamics with Physical Connection. Compared to individual MT dynamics, multiple MTs dynamics with physical connection is much complicated, because both translational and rotational movements are affected by neighboring forces, as shown in Figure 1(e). In this case, the translational dynamics can be written as

$$
M_{i} \dot{v}_{i}=F_{i}-\mu_{k} M g \frac{v}{\|v\|}-\sum_{k=1}^{K} \sum_{j=1}^{2} F_{i}^{k, j},
$$

where $K$ denotes the number of neighboring MTs, which affect current pushing manipulation, and $K \leq\left[F_{i} / \mu_{k} M g\right]$ should be satisfied. If only the neighbors are considered, then the equation can be simplified as follows:

$$
M_{i} \dot{v}_{i}=F_{i}-\mu_{k} M g \frac{v}{\|v\|}-\sum_{j=1}^{2}\left(F_{i}^{l, j}+F_{i}^{r, j}\right),
$$

where $F_{i}^{l, 1}, F_{i}^{l, 2}$ are the coupling forces at left-top and leftbottom corner of neighboring MT on the left side. $F_{i}^{r, 1}, F_{i}^{r, 2}$ are the coupling forces at right-top and right-bottom corner of neighboring MT on the right side. Compared with single MT, three types of torque are studied in this case, namely, kinetic frictional torque, HMSR's torque, and torques affected by neighboring MTs $T_{\text {no }}$. Thus, the overall rotational dynamics can be written as follows:

$$
\begin{aligned}
J_{i} \dot{\omega} & =T_{i}-T_{f}-T_{\text {no }} \\
& =F_{i} \times r_{i}-\frac{\mu_{k} g}{\left\|v_{a}\right\|} J_{i} \omega-\sum_{j=1}^{2}\left(F_{i}^{l, j} \times r_{i}^{l, j}+F_{i}^{r, j} \times r_{i}^{r, j}\right),
\end{aligned}
$$

where $r_{i}^{l, 1}$ and $r_{i}^{l, 2}$ are position vectors of left-top corner and left-bottom corner relative to mass center $x_{c}$ respectively. $r_{i}^{r, 1}$ and $r_{i}^{r, 2}$ are position vectors of right-top corner and rightbottom corner relative to mass center $x_{c}$ respectively. Since the shape of MT is centrosymmetric, $r_{i}^{l, j}=-r_{i}^{r, 2-j}(j=1,2)$. Thus, the third term of above equation can be rewritten as $\left(F_{i}^{r, 1}-F_{i}^{l, 2}\right) \times r_{i}^{r, 1}-\left(F_{i}^{l, 1}-F_{i}^{r, 2}\right) \times r_{i}^{l, 1}$.

2.3. Translational Control. The translational motion of MT is governed by the pushing leader of HMSRs, which knows the destination either by programmatic determination or manual guidance. Pushing leader's controller is designed to push its corresponding MT along the desired destination or trajectory by applying a force. It is quite simple as long as it reduces the error between the MT's actual velocity and desired velocity. The controller is chosen as follows:

$$
F_{i}^{\text {trans }}=K_{p} \max \left\{\left\|v_{i}^{d}\right\|-\left\|v_{i}\right\|, 0\right\} \frac{v_{i}^{d}}{\left\|v_{i}^{d}\right\|},
$$

where $v_{i}^{d}$ is the desired velocity and $K_{p}$ is the proportional factor of controller. From the above equation, $F_{i}^{i}$ has the same direction as the desired velocity. The magnitude of pushing force is adjusted by the difference between $v_{i}^{d}$ and $v_{i}$.

2.4. Rotational Control. Since MTs are physically connected with each other, and the rotational motion is governed by two neighboring MTs, which are also manipulated by neighboring HMSRs. Neighbor's force needs to overcome frictional forces corresponding to pushing leader and pushing followers. Then the pushing follower's controller can be derived as follows:

$$
F_{i}^{\mathrm{rot}}=\frac{1}{2}\left(\sum_{j=0}^{2} \mu_{k} M_{i} g \frac{v_{i+j-1}}{\left\|v_{i+j-1}\right\|}\right) .
$$

From this equation, we know that the rotational motion of $i$ th MT is affected by two neighbors. Because the neighboring MTs' velocity provides information for pushing follower's controller, the communication is necessary for rotational control in this multirobot system. This solution totally depends on communication between neighboring HMSR and may lead to control uncertainties when using unstable communication, especially in harsh environment. Considering that all connected MTs are pushed to align the same direction and velocity in the final state, thus, we can eliminate reliance on communication by letting $v_{i+j-1}^{t} \longrightarrow v_{i}^{t}$ $(j=0,2)$ when it achieves steady state, i.e., $t \longrightarrow+\infty$. 


\subsection{Convergence Proof of Pushing Dynamics}

Lemma 1. As proved in Wang's solution [11], given a constant vector $w$, a vector $v$ can be updated using the following discrete formula $v_{t+1}=\alpha_{t} \omega+\beta_{t} v_{t}$, where $\alpha_{t}$ is a series of nonnegative constants, and $\left\{\beta_{t} \mid 0<\beta_{t}<1\right\}$. Thus, the direction of $v_{t}$ will converge to the direction of $w$ at $t \longrightarrow+\infty$, i.e., $v_{t} \longrightarrow \gamma \omega$, where $\gamma=\alpha_{t-1}+\beta_{t-1} \alpha_{t-2}+\beta_{t-1} \beta_{t-2} \alpha_{t-3}+\cdots+\beta_{t-1} \beta_{t-2} \cdots$ $\beta_{1} \alpha_{0}$ is a positive scalar.

For pushing dynamic system described in equations (9) and (10), equation (7) can be rewritten as follows:

$$
\begin{aligned}
M_{i} \frac{v_{i}^{t+1}-v_{i}^{t}}{\Delta t} & =K_{p} \max \left\{\left\|v_{i}^{d}\right\|-\left\|v_{i}\right\|, 0\right\} \frac{v_{i}^{d}}{\left\|v_{i}^{d}\right\|}-\mu_{k} M_{i} g \frac{v_{i}^{t}}{\left\|v_{i}^{t}\right\|}-F_{i}^{\mathrm{rot}} \\
F_{i}^{\mathrm{rot}} & =\frac{1}{2}\left(\sum_{j=0}^{2} \mu_{k} M_{i} g \frac{v_{i+j-1}}{\left\|v_{i+j-1}\right\|}\right) .
\end{aligned}
$$

For simplification, the above equation can be rewritten as follows:

$$
\begin{aligned}
M_{i} \frac{v_{i}^{t+1}-v_{i}^{t}}{\Delta t}= & \frac{K_{p} \max \left\{\left\|v_{i}^{d}\right\|-\left\|v_{i}\right\|, 0\right\} v_{i}^{d}}{\left\|v_{i}^{d}\right\|} \\
& -\frac{1}{2} \mu_{k} M_{i} g\left(\frac{3 v_{i}^{t}}{\left\|v_{i}^{t}\right\|}+\frac{v_{i-1}^{t}}{\left\|v_{i-1}^{t}\right\|}+\frac{v_{i+1}^{t}}{\left\|v_{i+1}^{t}\right\|}\right)
\end{aligned}
$$

Since the purpose is to push all MTs with a specified formation aligning with the same direction, thus, the velocity difference should be minimized to zero, which means that the velocity of neighboring MT should be the same at $t \longrightarrow+\infty$, i.e.,

$$
\begin{aligned}
v_{i}^{t+1}-v_{i}^{t} & =\frac{\Delta t}{M_{i}} K_{p} \max \left\{\left\|v_{i}^{d}\right\|-\left\|v_{i}\right\|, 0\right\} \frac{v_{i}^{d}}{\left\|v_{i}^{d}\right\|}-\frac{5 \mu_{k} g \Delta t}{2} \frac{v_{i}^{t}}{\left\|v_{i}^{t}\right\|}, \\
v_{i}^{t+1} & =\frac{\Delta t K_{p} \max \left\{\left\|v_{i}^{d}\right\|-\left\|v_{i}\right\|, 0\right\}}{M_{i}\left\|v_{i}^{d}\right\|} v_{i}^{d}+\left(1-\frac{5 \Delta t \mu_{k} g}{2\left\|v_{i}^{t}\right\|}\right) v_{i}^{t} .
\end{aligned}
$$

Then, we can use the conclusion in Lemma 1 by letting

$$
\begin{array}{r}
\alpha_{t}=\frac{\Delta t K_{p} \max \left\{\left\|v_{i}^{d}\right\|-\left\|v_{i}\right\|, 0\right\}}{M_{i}\left\|v_{i}^{d}\right\|}, \\
\beta_{t}=\left(1-\frac{5 \Delta t \mu_{k} g}{2\left\|v_{i}^{t}\right\|}\right),
\end{array}
$$

which is true when the time step satisfies $0<$ $\Delta t<0.4\left\|v_{i}^{t}\right\| / \mu_{k} g$. Therefore, $v_{i}^{t}$ will converge to that of $v_{i}^{d}$ as well. The dynamics can be propagated further to the initial velocity $v_{i}^{0}$. Thus, $\left\|v_{i}^{t}-\gamma v_{i}^{d}\right\| \leq \beta_{m}^{t}\left\|v_{i}^{0}\right\|$ is true, where $\gamma=\alpha_{t-1}+\beta_{t-1} \alpha_{t-2}+\beta_{t-1} \beta_{t-2} \alpha_{t-3}+\cdots+\beta_{t-1} \beta_{t-2} \cdots \beta_{1} \alpha_{0}$, and $\beta_{m}^{t}=\max \left\{\beta_{0}, \beta_{1}, \ldots, \beta_{t-1}\right\}$. It can be concluded that $v_{i}^{t}$ converges to $\gamma v_{i}^{d}$ exponentially fast, and pushing followers' forces will converge to the pushing leader's force exponentially fast as well. Based on the above analysis, the pushing dynamic system can be established and integrated into the final virtual environment. Both the rotational and translational motions can be simulated through dynamic equations.

\section{Memory-Pushing Fuzzy Control Strategy}

For pushing manipulation of multiple sensorless HMSRs, pushing displacement along the $X$ axis is the main result after pushing manipulation. Theoretically, pushing displacement depends on pushing time because the hydraulic pressure maintains the same value. The longer pushing time is occupied, and the farther pushing displacement will be obtained. However, this work is extremely difficult for such a large-scale distributed and autonomous control system with sensorless characteristic and unstable communication. What is worse, the power of HMSR is supplied by hydraulic pump, which is mounted far away from the work field. There is a very long distance for emulsified liquid to pass through. As a result, the pressure loss is inevitable. Thus, it is very difficult to push all MTs to align with each other.

3.1. Time-Based Control Variable. As described above, the pressure loss is one of the main factors that affect pushing manipulation. Thus, the relation model between pressure loss and corresponding pushing time difference is studied in this section. The pressures between two adjacent HMSRs are different from each other. As shown in Figure 2(a), the details about hydraulic scheme of pushing control system are presented. Since the main concern of pressure loss is transportation length between HMSRs, then the frictional pressure loss is a key factor in this paper. Theoretically, the frictional pressure loss is proportional to the difference of pipeline length. Then, we can obtain the relation model as follows:

$$
\Delta p_{i}=p_{\text {in } i}-p_{\text {out } i}=\lambda \frac{L_{i}}{d_{i}} \times \frac{\rho^{2}}{2}=\frac{2 \lambda L_{i} \rho q_{i}}{\pi d_{i}^{3}},
$$

where $\lambda$ is the frictional pressure loss coefficient, $L_{i}$ is the $i$ th frictional pressure loss length between two adjacent HMSRs, $d_{i}$ is the $i$ th inner diameter of pipeline, $\rho$ is the density of the emulsified liquid, and $q_{i}$ is $i$ th primary flow rate of hydraulic pump. The inlet and outlet emulsified liquid pressures can be denoted as $P_{\text {in }}=\left[p_{\text {in } 1}, p_{\text {in } 2}, \ldots, p_{\text {in } N}\right]$ and $P_{\text {out }}=\left[p_{\text {out } 1}\right.$, $\left.p_{\text {out } 2}, \ldots, p_{\text {out } N}\right]$. As an independent and intelligent control unit, each HMSR is obstructed with external force $F_{i}$ and outputs pushing displacement $x\left(t_{i}\right)$. Due to frictional pressure loss, the elements in $P_{\text {in }}$ are different from each other. Thus, pushing times are different from each other as well.

To push MTs aligning at the same baseline, reference pushing time should be assigned to the pushing leader. The relationship between the pushing time difference and pressure difference can be denoted as follows:

$$
\Delta t_{i}=f\left(\Delta p_{i}\right)=f\left(\frac{2 \lambda L_{i} \rho q_{i}}{\pi d_{i}^{3}}\right)=f\left(k L_{i}\right),
$$

where $f(\cdot)$ is a nonlinear transfer function, which describes the relation between pushing time difference and pressure 


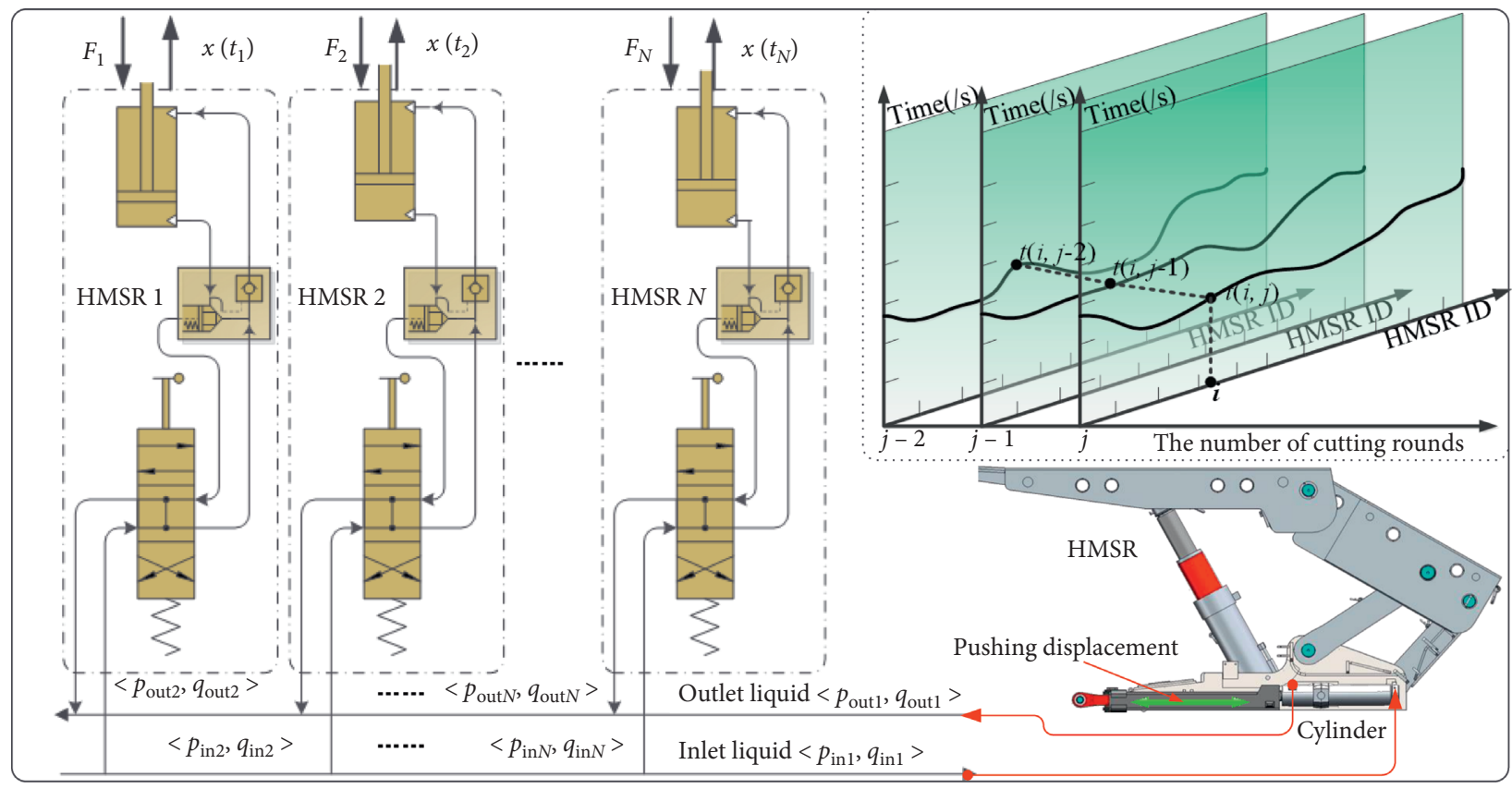

(a)

(b)

FIGURE 2: Memory-pushing system: (a) the hydraulic scheme of the pushing system; (b) time-based memory-pushing curve.

difference, and the detailed relation is difficult to be obtained. Statistic learning based predictors are one of solutions for this problem. However, this is beyond the scope of this paper, and it will be studied in future researches. In this paper, the function is simplified as $\Delta t_{i}=k L_{i}$, where the parameter $k$ is the linear coefficient, which can be obtained from the field experiment. Then, the reference pushing time for $i$ th HMSR can be calculated as follows:

$$
t_{i}^{R}=t_{0}^{R}+\sum_{m=1}^{i} k L_{m}
$$

where $t_{0}^{R}$ is the required pushing time to finish the pushing manipulation without delay, which is affected by frictional pressure loss.

$$
T=\left\{\begin{array}{cccc}
{[t(1,1)} & t(1,2) & \ldots & t(1, j)] \\
{[t(2,1)} & t(2,2) & \ldots & t(2, j)] \\
{[\ldots} & \ldots & \ldots & \ldots] \\
{[t(i, 1)} & t(i, 2) & \ldots & t(i, j)]
\end{array}\right\}
$$

where $N$ denotes the total number of HMSR, and $m$ is the finite number of cutting round. The time-based memorypushing curve is shown in Figure 2(b). From the figure, for the same HMSR, in different cutting round, the pushing time should be adjusted according to the previous round of cutting. The variables are pushing time difference and pushing time difference rate, which are presented as $E\left(t_{i}\right)$ and $\Delta E\left(t_{i}\right)$, respectively. They are calculated as follows:
3.2. Memory-Pushing Model. Pushing manipulation is a tough work due to the sensorless and noncommunicative characteristics, and the pushing displacement $x\left(t_{i}\right)$ depends on pushing time $t_{i}$. Thus, a memory-pushing model based on the previous cycle of pushing manipulation is proposed for the system. To describe the model, the time-based memorypushing curve should be demonstrated at first. In each round of cutting, every HMSR will get a pushing time, which is marked as $t(i, j)$, where $i$ denotes the identify number of HMSRs, and $j$ denotes the $j$ th round of cutting. The information obtained from control system can be described as follows:

$(i=1,2,3, \ldots, N, j=1,2,3, \ldots, m)$

$$
\begin{aligned}
E\left(t_{i}\right) & =t(i, j)-t_{i}^{R}, \\
\Delta E\left(t_{i}\right) & =\frac{\mathrm{d}\left[E\left(t_{i}\right)\right]}{\mathrm{d} t} \approx E\left(t_{i}\right)-E\left(t_{i-1}\right) .
\end{aligned}
$$

According to above equation, we know that the reference time $t_{i}^{R}$ is fixed for a specified HMSR, no matter how cutting round $j$ changes. Then the equation can be transformed as follows: 


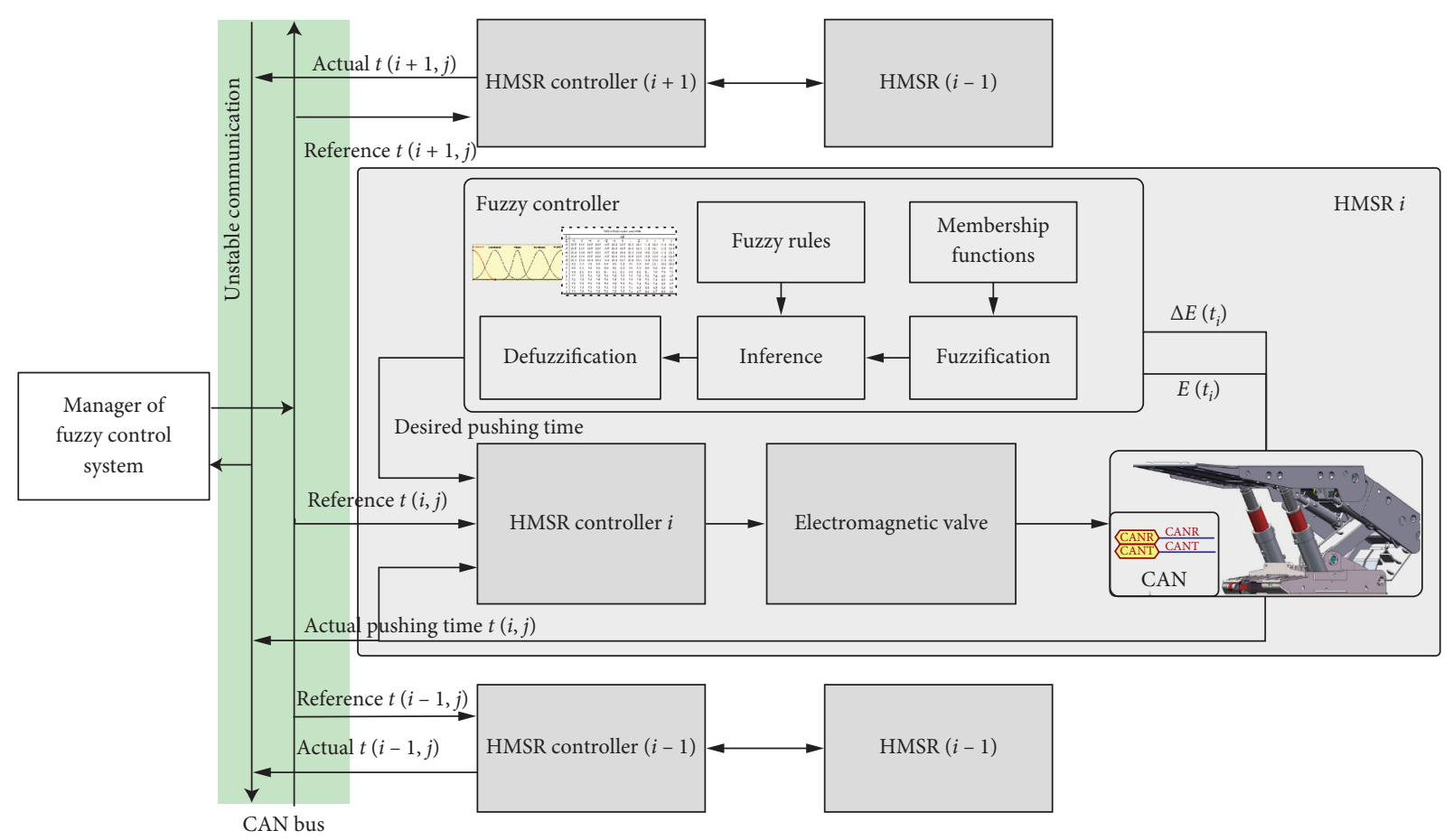

FIGURE 3: Structure of memory-pushing fuzzy control approach; the unstable communication based on CAN bus is only used for specifying the reference pushing time and sending the start command.

$$
\begin{gathered}
E\left(t_{i}\right)=t(i, j)-t_{0}^{R}+\sum_{m=1}^{i} k L_{m}, \\
\Delta E\left(t_{i}\right)=\frac{\mathrm{d}\left[E\left(t_{i}\right)\right]}{\mathrm{d} t} \approx E\left(t_{i}\right)-E\left(t_{i-1}\right) .
\end{gathered}
$$

The control objective is to adjust current pushing time and make it approximate to the corresponding reference pushing time $t_{i}^{R}$ for $i$ th HMSR. The control variable is designed based on the above equations as well.

3.3. Memory-Pushing Fuzzy Controller. Due to the inaccuracy of pushing manipulation for sensorless HMSR, fuzzy control strategy is applied into this system. The workflow of fuzzy controller can be described as follows:

(1) Memory-pushing time acquisition: in order to satisfy the requirements of memory-pushing control, the actual pushing time of the previous cutting round and the reference pushing time will be recorded or assigned programmatically, and the desired pushing time will be calculated with the help of memorypushing time.

(2) Input variable determination: the time difference between the actual pushing time and reference pushing time $E\left(t_{i}\right)$ and the time difference rate $\Delta E\left(t_{i}\right)$ are considered as the input variables for the fuzzy control system. The input variable pushing time difference and pushing time difference rate can be calculated according equation (19) or equation (20).
(3) Fuzzy universe and fuzzy set determination: the variable $\left\{E\left(t_{i}\right), \Delta E\left(t_{i}\right)\right\}$ is set based on the actual variation range. And the corresponding linguistic variable can be set in order to describe problem distinctly. For output variable, the actual boundary of control variables $[A, B]$ will be limited to a certain range $[a, b]$, and the converted equation in this paper is given as follows:

$$
Y=\frac{B-A}{b-a}\left(X-\frac{a+b}{2}\right) .
$$

(4) Fuzzy membership function, fuzzy rule, and fuzzy control query table: in order to improve the index fuzziness, the fuzzy membership function is often set to gaussmf function, and the function can be calculated as follows:

$$
\text { gaussmf }=e^{-(x-c)^{2} / 2 \sigma^{2}},
$$

With help of linguistic variable, the fuzzy rule can be established distinctly. For time difference $E$, the fuzzy set is $\{\mathrm{NL}$, NM, S, PM, PL\} which represents ["Time largely decreased," "Time is mezzo," "Time increased," Time largely increased"\}. For time difference rate $\Delta E$, the fuzzy set is $\{\mathrm{NL}, \mathrm{NM}, \mathrm{S}, \mathrm{PM}, \mathrm{PL}\}$ which represents ["Time decreased very fast," "Time decreased faster," "Time difference rate is constant," "Time increased faster," "Time increased very fast" . According to these linguistic variable definition and fuzzy rules, control system processes a lot of fuzzy operations and sets a series of firing strength. Finally, the fuzzy control query table can be calculated, and the 


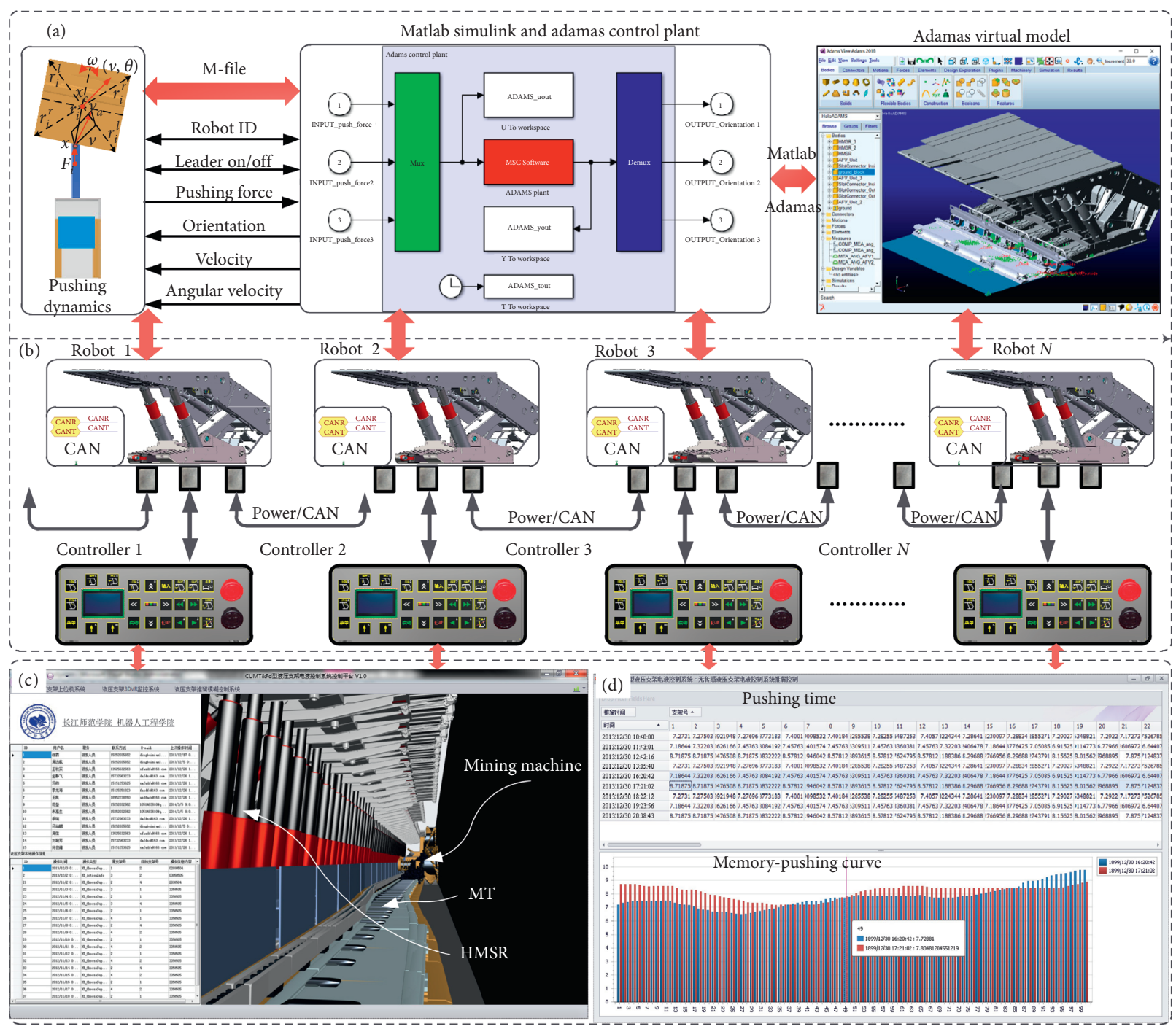

FIGURE 4: System architecture: (a) the virtual simulation environment for noncommunicative memory-pushing fuzzy control system; (b) hardware architecture of existing multirobot system; (c) user interface of proposed control system; (d) data visualization of proposed control system.

fuzzy control system can be integrated into the proposed fuzzy memory-pushing control system. The structure of memorypushing fuzzy control approach is shown in Figure 3. Each HMSR has a controller, and for each HMSR controller, the reference pushing time is specified by the control manager via CAN Bus or guided by human. Based on the reference pushing time and the last round of actual pushing time, the fuzzy controller with 2 input variables, i.e., $E\left(t_{i}\right)$ and $\Delta E\left(t_{i}\right)$, is capable of obtaining the next round of pushing time. Finally, the current HMSR controller can drive the electromagnetic value to push MT forward.

For a specified HMSR, the pushing manipulation begins when pushing command is received via Controller Area Network (CAN) bus. Firstly, the memory-pushing time of the $j$ th and $(j-1)$ th round of cutting, namely, $t(i, j)$ and $t(i, j-1)$, and the current reference pushing time will be confirmed. Secondly, the designated input parameters will be transmitted to fuzzy controller; the fuzzy controller calculates the appropriate pushing time according to the fuzzy control query table. Thirdly, the output pushing time from fuzzy controller will be compared with the actual pushing time $t(i, j+1)$. Because the maximum pushing length is limited by physical connections between neighboring MTs, HMSR will push corresponding MT until the torques between neighbors approximate to zero. If $\| t(i, j)-$ $t(i, j+1) \| \leq$ Maximum then the current pushing time will be memorized, while the pushing obstacles can be detected if $\|t(i, j)-t(i, j+1)\|>$ Maximum, and the conflict message will be transmitted to manager of fuzzy control system or operators. And the pushing manipulation can be terminated or reset. By executing such work cycle repeatedly, the memory-pushing fuzzy control process can be completed successfully.

\section{Simulation and Experiment}

4.1. Architecture. The proposed noncommunicative memory-pushing fuzzy control strategy is simulated in a threelayer architecture based virtual simulation environment, as shown in Figure 4(a). Matlab Simulink and Adams Control 


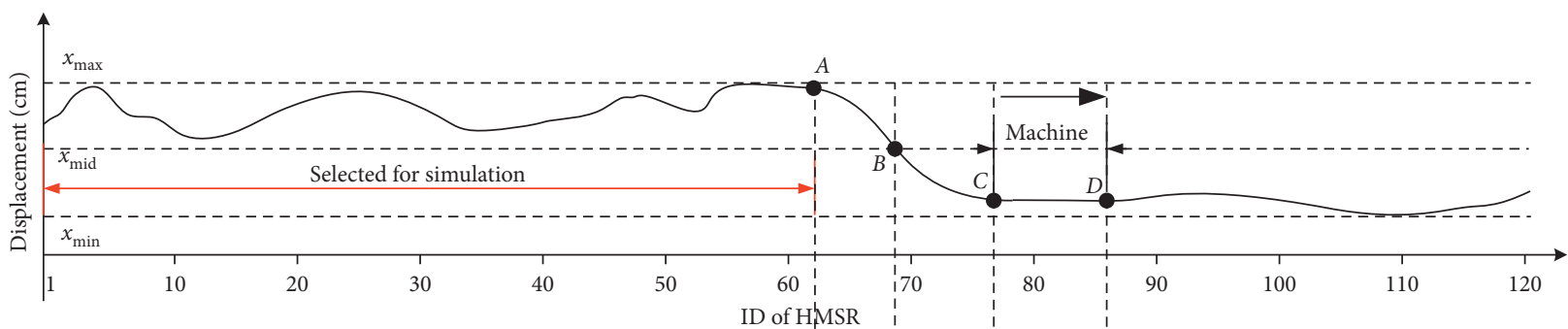

(a)

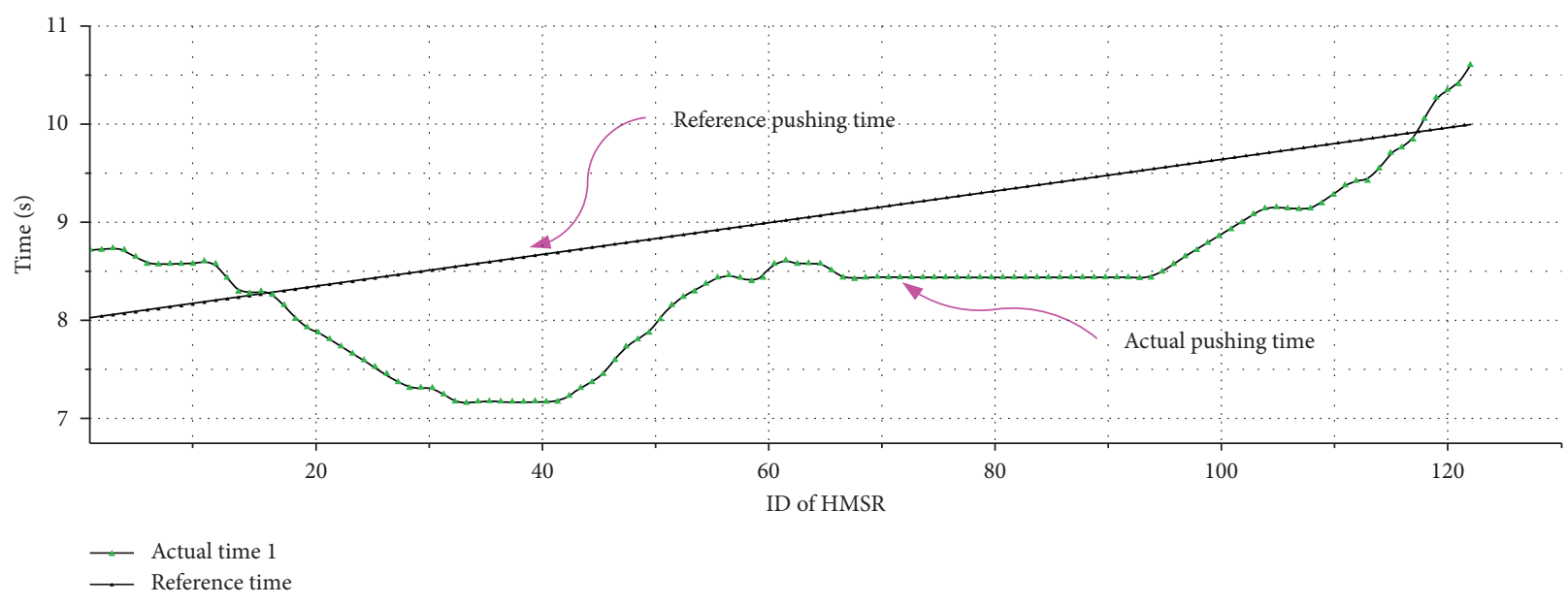

(b)

FIgURE 5: Pushing displacement and pushing time of testing HMSRs, the abscissa axis represents the ID of HMSR; (a) pushing displacement of current status; (b) memory-pushing time in the last round of cutting.

are utilized for establishing the sensorless multirobot system. We built the simplified dynamic system by using the realscale 3D parametrical model of HMSRs and MTs, which are demonstrated in Figure 4(b). The attachment point between HMSR and MT is simplified as 2-DOF revolute joint, and the physical connections between MTs are built based on the collision detection between rigid bodies. Then the established Adams virtual model can be invoked in Matlab Simulink through Adams control plugin. The output Adams control plant provides data interfaces to Simulink. Those interfaces are listed as follows:

(1) Robot ID, unique identity number corresponding to the index of HMSR

(2) Leader on/off, a Boolean variable that indicates whether the HMSR is a pushing leader or follower

(3) Pushing force, float-type value that is denoted as the pushing force of HMSR

(4) Orientation, velocity, and angular velocity are the pose information about MTs, which can be measured by IMU

Based on these data interfaces, pushing dynamics can be integrated into the virtual simulation environment, and the memory-pushing fuzzy controller can be implemented using Matlab. However, we can notice that hydraulic part is ignored in the virtual environment, because it is only concerned with pushing pressure loss, which is compensated in the memory-pushing fuzzy controller. Finally, the simulation can be conducted with the established environment.

A GUI system is also developed for the proposed control system, which utilizes data from proposed control system through Ethernet. As shown in Figures 4(c) and 4(d), the main user interface of the proposed system and the data visualization of memory-pushing fuzzy control system are presented, respectively. Under the main user interface, all user information, user operation history, and $3 \mathrm{D}$ virtual monitoring interface are demonstrated. All the pushing operations about HMSR can be displayed in real time. Under the data visualization interface, all pushing parameters such as current pushing time, history pushing time, and execution time are presented. Moreover, the pushing time of each HMSR will be presented as memory-pushing curve in the bar chart real-timely.

4.2. Simulation. In this section, an underground fully mechanized coal mining face with 122 sensorless HMSRs, which are identified with a unique integer value, i.e., ID of HMSR, is selected as the test sample. Current status, i.e., pushing displacement and pushing time, of these HMSRs is shown in Figure 5(a), and pushing time was obtained as shown in Figure 5(b). As mentioned previously, due to system pressure loss, the reference pushing time is a slant line. HMSR will suffer more pressure loss if it is closer to the 
TAble 1: Pushing displacement of the segmentation selected for simulation $(\mathrm{mm})$.

\begin{tabular}{|c|c|}
\hline ID & Distance \\
\hline 1 & 778.5 \\
\hline 2 & 765 \\
\hline 3 & 752.3 \\
\hline 4 & 739.5 \\
\hline 5 & 726 \\
\hline 6 & 713.2 \\
\hline 7 & 703.5 \\
\hline 8 & 694.8 \\
\hline 9 & 687.9 \\
\hline 10 & 687.9 \\
\hline 11 & 687.9 \\
\hline 12 & 687.9 \\
\hline 13 & 695.7 \\
\hline 14 & 702.7 \\
\hline 15 & 708.1 \\
\hline 16 & 721.2 \\
\hline 17 & 727 \\
\hline 18 & 739.3 \\
\hline 19 & 744.6 \\
\hline 20 & 750.4 \\
\hline 21 & 758.2 \\
\hline 22 & 766.1 \\
\hline 23 & 766.1 \\
\hline 24 & 766.1 \\
\hline 25 & 758.2 \\
\hline 26 & 752.5 \\
\hline 27 & 747.4 \\
\hline 28 & 734.6 \\
\hline 29 & 715.7 \\
\hline 30 & 696.9 \\
\hline 31 & 678.3 \\
\hline 32 & 658 \\
\hline 33 & 641.4 \\
\hline 34 & 625.4 \\
\hline 35 & 619 \\
\hline 36 & 617.5 \\
\hline 37 & 618.3 \\
\hline 38 & 631 \\
\hline 39 & 639.3 \\
\hline 40 & 657.3 \\
\hline 41 & 670 \\
\hline 42 & 691.4 \\
\hline 43 & 712 \\
\hline 44 & 725.4 \\
\hline 45 & 746.4 \\
\hline 46 & 759.5 \\
\hline 47 & 772.3 \\
\hline 48 & 777.6 \\
\hline 49 & 789.5 \\
\hline 50 & 789.5 \\
\hline 51 & 781.7 \\
\hline 52 & 778.2 \\
\hline 53 & 766.1 \\
\hline 54 & 753.8 \\
\hline 55 & 735 \\
\hline 56 & 716.5 \\
\hline 57 & 689.7 \\
\hline 58 & 680.1 \\
\hline 59 & 682.1 \\
\hline 60 & 694.8 \\
\hline
\end{tabular}

TABle 1: Continued.

\begin{tabular}{lc}
\hline ID & Distance \\
\hline 61 & 733.7 \\
62 & 763.7 \\
63 & 789.5 \\
64 & 789.5 \\
65 & 789.5 \\
66 & 789.5 \\
67 & 789.5 \\
\hline
\end{tabular}

right side, and the longer pushing time will be consumed. Assume that the space between HMSRs is a constant value and the linear coefficient is known as an empirical value, i.e., $k=0.0164$. Then the reference pushing time of $i$ th HMSR can be obtained as $t_{i}^{R}=t_{0}^{R}+1.5 k \cdot i$.

For the purpose of demonstrating the entire process of pushing manipulation, we chose the most undulating segment of the pushing curve, which has been highlighted in red color in Figure 5(a), and the details of pushing displacement are shown in Table 1.

Actually, because of terrible work environment, different geological conditions, and frictional pressure loss, the actual pushing time is a curve with partial fluctuations. In the fuzzy control system, the pushing time difference $E\left(t_{i}\right)$ and the pushing time difference rate $\Delta E\left(t_{i}\right)$ are considered as the input variables. These input variables can be calculated with equation (19). The results are shown in Figure 6. The variation range of pushing time difference $E\left(t_{i}\right)$ is set to $[-2,2]$, universe is set to $[-6,6]$, and fuzzy set is $\{\mathrm{NL}, \mathrm{NM}, \mathrm{S}, \mathrm{PM}$, $\mathrm{PL}$. The variation range of pushing time difference rate $\Delta E\left(t_{i}\right)$ is set to $[-2,2]$ as well, and universe is set to $[-6,6]$. For the output variable $U$, the variation range is from 6 to 12 . The actual boundary of the variables can be calculated by equation (21).

Based on the above definition, the fuzzy controller can be implemented using Matlab. With the help of linguistic variable, the fuzzy rule can be made distinctly. According to these fuzzy rules, the control system will process a lot of fuzzy operations and set a serious of firing strength. Finally, the fuzzy control query table can be obtained, and details are shown in Table 2.

Once the fuzzy control query table is confirmed, the fuzzy control system can be integrated into our memorypushing fuzzy control system. Three typical results are recorded using the proposed data visualization system; results are shown in Figure 7.

In this simulation, only 67 HMSRs are selected for the demonstration of pushing manipulation, in the first stage of pushing manipulation, as shown in Figure 7(a), the 10th, $35^{\text {th }}$, and 58th HMSRs are programmatically assigned with pushing destination according to the sum of pushing displacements left to be pushed. Considering that the driven capability of hydraulic pump is limited, pushing operations are separated into several sequences in a specified pushing manipulation stage. Also, we assume that the hydraulic pump can provide enough pressure for at least 8 HMSRs at the same time. Then, the first pushing sequence in the first 


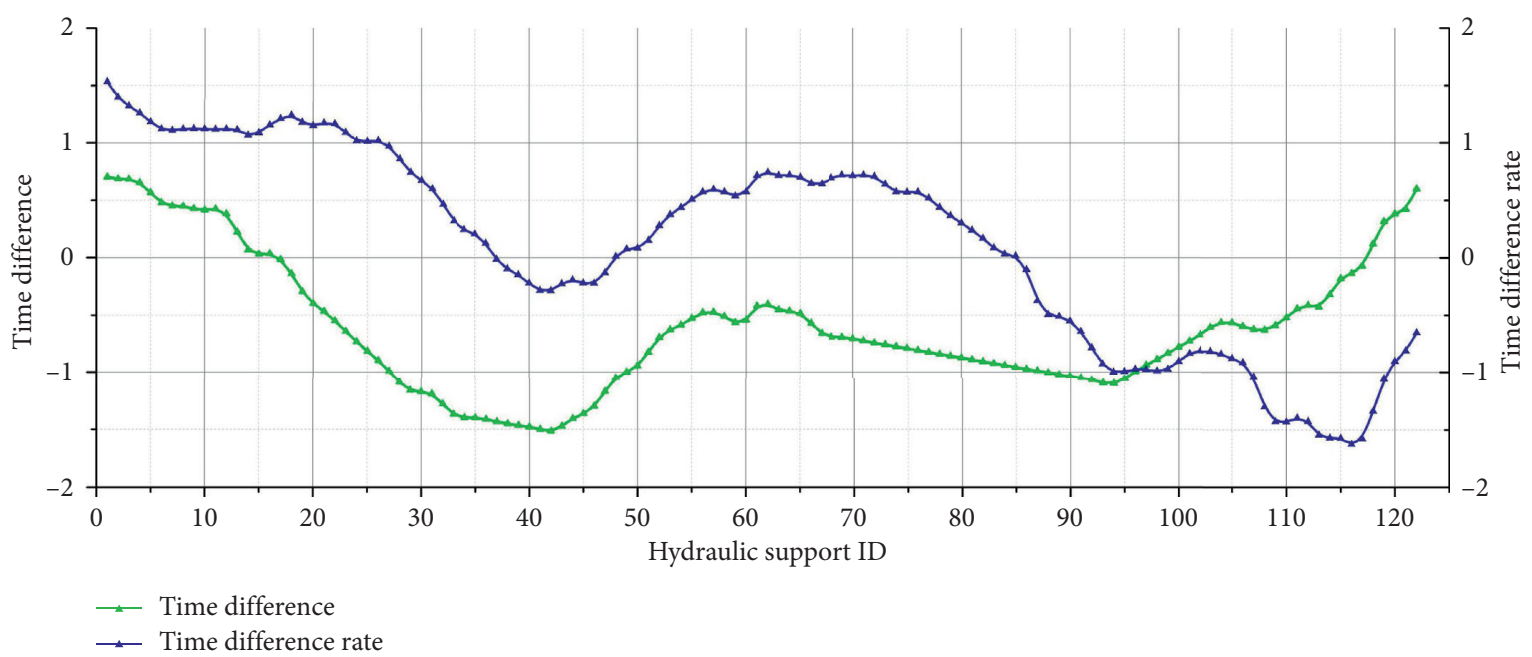

Figure 6: Pushing time difference and pushing time difference rate.

TABLe 2: Fuzzy control query table.

\begin{tabular}{|c|c|c|c|c|c|c|c|c|c|c|c|c|c|}
\hline$U$ & & & & & & & $\Delta E\left(t_{i}\right)$ & & & & & & \\
\hline$E\left(t_{i}\right)$ & -6 & -5 & -4 & -3 & -2 & -1 & 0 & 1 & 2 & 3 & 4 & 5 & 6 \\
\hline-6 & 10.5 & 10.5 & 10.5 & 10.5 & 10.5 & 10.4 & 10.5 & 10.6 & 11.3 & 11.4 & 11.3 & 11.4 & 11.4 \\
\hline-5 & 10.5 & 10.5 & 10.5 & 10.5 & 10.5 & 10.4 & 10.5 & 10.6 & 11.1 & 11.2 & 11.1 & 11.2 & 11.4 \\
\hline-4 & 10.4 & 10.4 & 10.5 & 10.5 & 10.5 & 10.4 & 10.4 & 10.6 & 10.6 & 10.6 & 10.6 & 11.1 & 11.3 \\
\hline-3 & 10.4 & 10.4 & 10.5 & 10.5 & 10.5 & 10.4 & 10.4 & 10.4 & 10.5 & 10.5 & 10.6 & 11.2 & 11.4 \\
\hline-2 & 10.3 & 10.4 & 10.4 & 10.4 & 10.4 & 10.4 & 10.3 & 10.4 & 10.4 & 10.4 & 10.6 & 11.1 & 11.3 \\
\hline-1 & 9.2 & 9.9 & 9.9 & 9.9 & 9.9 & 9.9 & 9.2 & 9.9 & 9.9 & 9.9 & 10.1 & 10.1 & 10.1 \\
\hline 0 & 9.0 & 9.0 & 9.0 & 9.0 & 9.0 & 9.0 & 9.0 & 9.0 & 9.0 & 9.0 & 9.0 & 9.0 & 9.0 \\
\hline 1 & 8.8 & 8.1 & 8.1 & 8.1 & 8.1 & 8.1 & 8.1 & 8.1 & 8.1 & 8.1 & 7.9 & 7.9 & 7.9 \\
\hline 2 & 7.7 & 7.5 & 7.5 & 7.5 & 7.5 & 7.5 & 7.5 & 7.5 & 7.5 & 7.5 & 7.4 & 6.9 & 6.7 \\
\hline 3 & 7.6 & 7.5 & 7.5 & 7.5 & 7.5 & 7.5 & 7.5 & 7.5 & 7.5 & 7.5 & 7.4 & 6.8 & 6.6 \\
\hline 4 & 7.5 & 7.5 & 7.5 & 7.5 & 7.5 & 7.5 & 7.5 & 7.4 & 7.4 & 7.4 & 7.4 & 6.9 & 6.7 \\
\hline 5 & 7.5 & 7.5 & 7.5 & 7.5 & 7.5 & 7.5 & 7.5 & 7.4 & 6.9 & 6.8 & 6.9 & 6.8 & 6.6 \\
\hline 6 & 7.5 & 7.5 & 7.5 & 7.5 & 7.5 & 7.5 & 7.5 & 7.4 & 6.7 & 6.6 & 6.7 & 6.6 & 6.6 \\
\hline
\end{tabular}

pushing manipulation stage needs 5 steps of pushing, $18.7 \mathrm{~s}$ in total. The second and third pushing sequences cost 5 steps (11.4s) and 3 steps (11.3s), respectively.

Based on the above pushing result, in the second stage of pushing manipulation, there are more pushing sequences that need to be pushed. The most complex pushing process is the second pushing sequence, which needs 7 pushing steps, as shown in Figure 7(b). However, it only needs $12 \mathrm{~s}$ compared with the first pushing sequence $(13.3 \mathrm{~s})$. The simulation result fits our cognition about pushing operation; i.e., the more pushing displacement left, the longer pushing time will be consumed. But this is not absolutely right, because the proposed system is totally autonomous and noncommunicative, which means that the cooperation between HMSRs also affects the final pushing result. This is verified by the pushing results of 5 th and 6th pushing sequences. Theoretically, there should be more pushing displacement left for the 5th pushing sequence, then more pushing time should be occupied, while only $2.6 \mathrm{~s}$ is the cost for the 5 th pushing sequence and $3.2 \mathrm{~s}$ for the 6 th pushing sequence. Thus, if the cooperation between HMSRs is good enough, much less pushing time will be occupied.

The details about pushing results and corresponding pushing time of the whole pushing process are shown in Table 3. The traditional human-guided manipulation needs to push MTs repeatedly, which may cost half an hour or even longer. What is worse, the pushing time and pushing steps are not predictable due to physical connections and limitations, and the operational uncertainties of human-guided process, while the whole process manipulated by the proposed system only costs 32 steps and $91.6 \mathrm{~s}$, which dramatically improves efficiency of pushing manipulation.

4.3. Industrial Experiment. The proposed memory-pushing fuzzy control system has been developed and applied in a coal mining in Shan Xi, China. For concerns of safety, we conducted this experiment for only one cutting round. In case of practical application without memory-pushing control, it is very difficult to push scraper conveyer to align with others, and the final pushing results totally depend on operators' repeated 


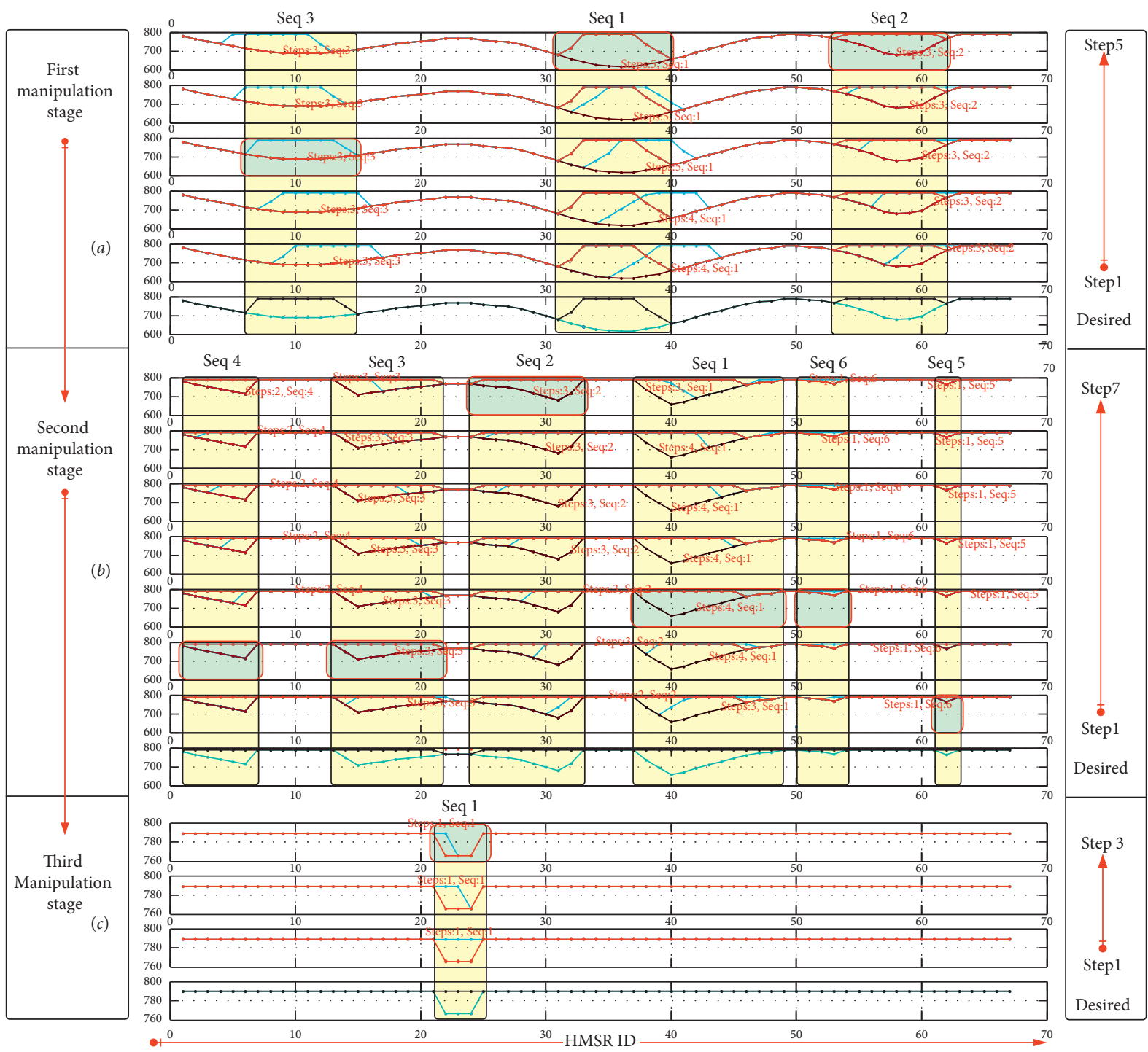

FIGURE 7: Simulation results: (a) the pushing result of first manipulation stage, three sequences of pushing manipulations are presented; (b) the pushing result of second manipulation stage, six sequences of pushing manipulations are presented; (c) the pushing result of third manipulation stage, only one sequence of pushing manipulation is presented. The bottom subplot in each manipulation stage shows the desired pushing status and current pushing status.

TABle 3: Pushing results of the simulation.

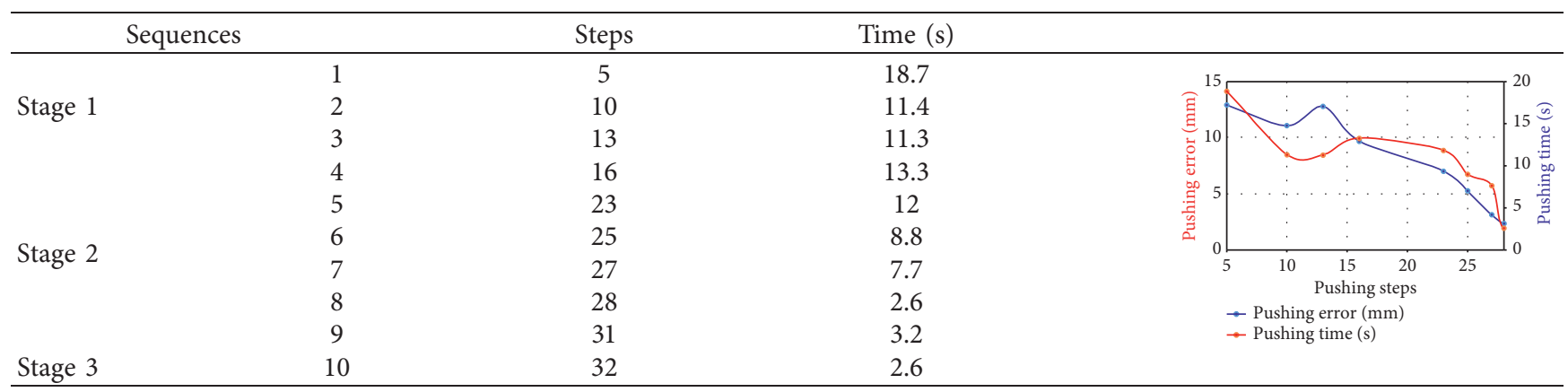

working. The photograph of work field is shown in Figure 8(a). Then, memory-pushing fuzzy control system is used to solve this problem, and the photograph is shown in Figure 8(b).
From the comparison between the two pictures, obviously, the pushing result of practical application with memory-pushing control strategy is much better. 


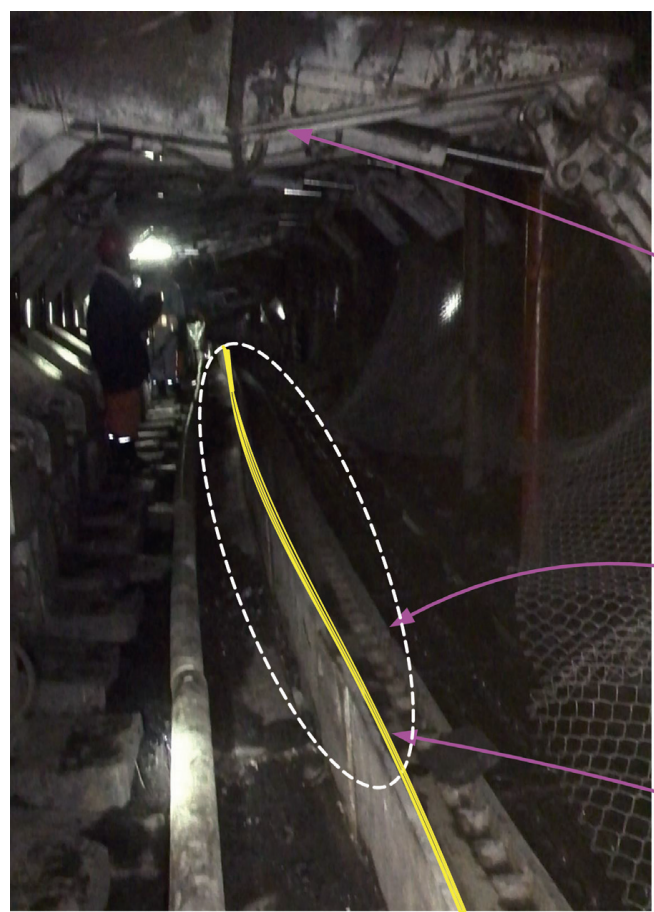

(a)

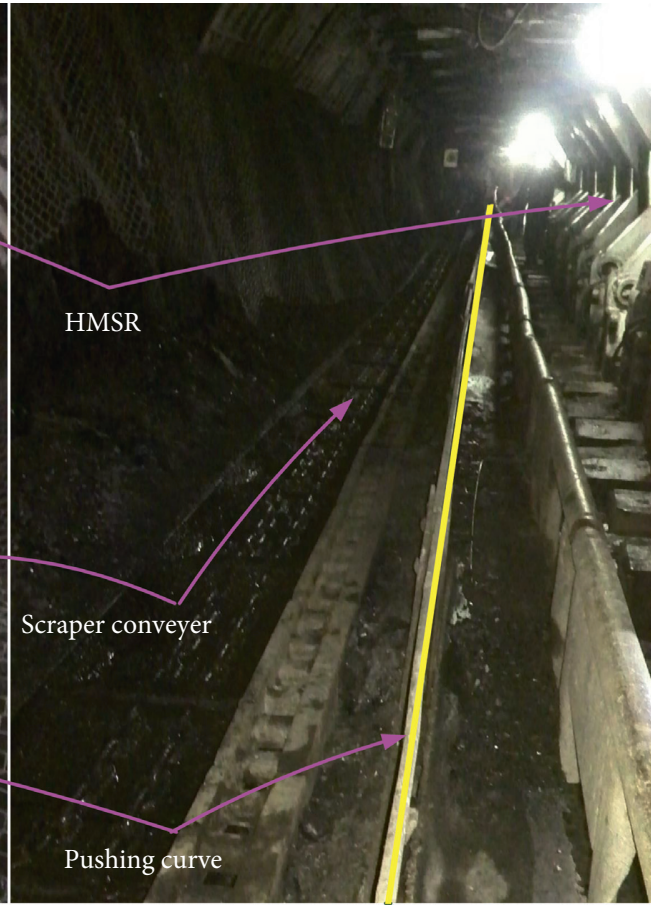

(b)

Figure 8: Industrial experiment. (a) Practical application without memory pushing control strategy. (b) Practical application with memory pushing control strategy.

\section{Conclusion}

To effectively and cooperatively push MT to align with each other, the problem of noncommunicative pushing manipulation of multirobot system, which takes HMSR as the pushing robot and MT as the manipulated object, is studied. The pushing dynamics of system is derived, and the convergence of pushing dynamics is proved considering noncommunicative situation. A time-based memory-pushing fuzzy control strategy is proposed and tested in the Adams based virtual environment, which integrates the derived pushing dynamics. Finally, the industrial experiment is demonstrated with only one cutting round example. Both the simulation and application results show that the proposed method can efficiently push MTs to align with each other within seconds, which saves a lot of time compared with traditional human-guided method. What is more, the method is totally distributed and autonomously dominated, which means that the system is still available when communication is disabled. Thus, the system is very feasible and efficient for practical application. As to future work, the proposed method will be further optimized; the relation between pushing time difference and pressure difference should be optimized.

\section{Data Availability}

The data used to support the findings of this study are included within the article.

\section{Conflicts of Interest}

The authors declare no conflicts of interest.

\section{Authors' Contributions}

Lin Zhang conceived and designed the experiments; Xianhua Zheng and Shang Feng performed the experiments; Lin Zhang and Lingling Su built the pushing dynamics; Shang Feng analyzed the data; Lin Zhang wrote the paper. Shang Feng translated and reedited the manuscript.

\section{Acknowledgments}

The support of Ministry of Education's Cooperative Education Project (No: 201902040072 and No: JG2020206), Science and Technology Research Program of Chongqing Municipal Education Commission (No: KJ1712312), Changzhou Science and Technology Project (No: CE20185040), and the project funded by Water Resources Department of Jiangsu Province (No:2018031) in carrying out this research is gratefully acknowledged.

\section{References}

[1] M. Sitti, "Robotic collectives inspired by biological cells," Nature, vol. 567, no. 7748, pp. 314-315, 2019.

[2] S. Won, S. Kim, J. E. Park, J. Jeon, and J. J. Wie, “On-demand orbital maneuver of multiple soft robots via hierarchical magnetomotility," Nature Communications, vol. 10, no. 1, pp. 1-8, 2019.

[3] A. Dussutour, V. Fourcassié, D. Helbing, and J.-L. Deneubourg, "Optimal traffic organization in ants under crowded conditions," Nature, vol. 428, no. 6978, pp. 70-73, 2004. 
[4] D. T. Tambe, C. Corey Hardin, T. E. Angelini et al., "Collective cell guidance by cooperative intercellular forces," Nature Materials, vol. 10, no. 6, pp. 469-475, 2011.

[5] S. Li, R. Batra, D. Brown et al., "Particle robotics based on statistical mechanics of loosely coupled components," Nature, vol. 567, no. 7748, pp. 361-365, 2019.

[6] H. F. McCreery and M. D. Breed, "Cooperative transport in ants: a review of proximate mechanisms," Insectes Sociaux, vol. 61, no. 2, pp. 99-110, 2014.

[7] A. Gelblum, I. Pinkoviezky, E. Fonio, A. Ghosh, N. Gov, and O. Feinerman, "Ant groups optimally amplify the effect of transiently informed individuals," Nature Communications, vol. 6, no. 1, p. 7729, 2015.

[8] O. Feinerman, I. Pinkoviezky, A. Gelblum, E. Fonio, and N. S. Gov, "The physics of cooperative transport in groups of ants," Nature Physics, vol. 14, no. 7, pp. 683-693, 2018.

[9] Z. Wang and M. Schwager, "Multi-robot manipulation without communication," in Distributed Autonomous Robotic Systems, vol. 112, pp. 135-149, Springer, Berlin, Germany, 2016.

[10] Z. Wang and M. Schwager, "Multi-robot manipulation with No communication using only local measurements," in Proceedings of the 2015 54th IEEE Conference on Decision and Control (CDC), IEEE, Osaka, Japan, pp. 380-385, December 2015.

[11] Z. Wang and M. Schwager, "Force-amplifying N-robot transport system (Force-ANTS) for cooperative planar manipulation without communication," The International Journal of Robotics Research, vol. 35, no. 13, pp. 1564-1586, 2016.

[12] N. Rezzoug and P. Gorce, "Dynamic control of pushing operations," Robotica, vol. 17, no. 6, pp. 613-620, 1999.

[13] M. Halm and M. Posa, "A quasi-static model and simulation approach for pushing, grasping, and jamming," in Proceedings of the International Workshop on the Algorithmic Foundations of Robotics, Springer, Merida, Mexico, pp. 491-507, December 2018.

[14] J. Pliego-Jimenez and M. Arteaga-Perez, "On the adaptive control of cooperative robots with time-variant holonomic constraints," International Journal of Adaptive Control and Signal Processing, vol. 31, no. 8, pp. 1217-1231, 2017.

[15] D. Ma and A. Rodriguez, "Friction variability in planar pushing data: anisotropic friction and data-collection bias," IEEE Robotics and Automation Letters, vol. 3, no. 4, pp. 3232-3239, 2018.

[16] W. C. Agboh and M. R. Dogar, "Pushing fast and slow: taskadaptive planning for non-prehensile manipulation under uncertainty," in Intelligent Technologies and Robotics, pp. 160-176, Springer, Cham, Switzerland, 2020.

[17] J.-L. Lin, "Adaptive reinforcement learning in box-pushing robots," International Journal of Automation and Smart Technology, vol. 8, no. 1, pp. 41-49, 2018.

[18] M. Rahimi, S. Gibb, Y. Shen, and M. L. Hung, "A comparison of various approaches to reinforcement learning algorithms for multi-robot box pushing," in Advances in Engineering Research and Application, pp. 16-30, Springer, Cham, Switzerland, 2019.

[19] K. Harada, J. Nishiyama, Y. Murakami, and M. Kaneko, "Pushing manipulation for multiple objects," Journal of Dynamic Systems, Measurement, and Control, vol. 128, no. 2, pp. 422-427, 2006.

[20] Z. Zyada, Y. Hayakawa, and S. Hosose, "Kinematic analysis of a two-link object for whole arm manipulation," in Proceedings of the 9th WSEAS International Conference on Signal
Processing, Robotics and Automation, pp. 139-145, Cambridge, UK, February 2010.

[21] B. Moldovan, P. Moreno, D. Nitti, J. Santos-Victor, and L. De Raedt, "Relational affordances for multiple-object manipulation," Autonomous Robots, vol. 42, no. 1, pp. 19-44, 2018.

[22] A. J. Shah and J. A. Shah, "Towards manipulation planning for multiple interlinked deformable linear objects," in Proceedings of the IEEE International Conference on Robotics and Automation, pp. 3908-3915, Stockholm, Sweden, June 2016.

[23] A. Shah, L. Blumberg, and J. Shah, "Planning for manipulation of interlinked deformable linear objects with applications to aircraft assembly," IEEE Transactions on Automation Science and Engineering, vol. 15, no. 4, pp. 1823-1838, 2018.

[24] E. Kamal, A. Aitouche, R. Ghorbani, and M. Bayart, "Fuzzy scheduler fault-tolerant control for wind energy conversion systems," IEEE Transactions on Control Systems Technology, vol. 22, no. 1, pp. 119-131, 2014.

[25] S. Grosswindhager, L. Haffner, A. Voigt, and M. Kozek, "Fuzzy modelling of a moving grate biomass furnace for simulation and control purposes," Mathematical and Computer Modelling of Dynamical Systems, vol. 20, no. 2, pp. 194-208, 2014.

[26] L. Zhang, X. Chang, and H. R. Karimi, "Fuzzy modeling and control for a class of inverted pendulum system," Abstract and Applied Analysis, vol. 2014, Article ID 936868, 6 pages, 2014.

[27] E. Kamal, A. Aitouche, and D. Abbes, "Robust fuzzy scheduler fault tolerant control of wind energy systems subject to sensor and actuator faults," International Journal of Electrical Power \& Energy Systems, vol. 55, pp. 402-419, 2014.

[28] P. Alvarez, B. McElwain, L. Thesing, S. Edalath, A. Kukreti, and K. Cohen, "PD and fuzzy logic control for earthquake resilient structures," Computer Applications in Engineering Education, vol. 22, no. 1, pp. 142-152, 2014.

[29] S. Tong, S. Sui, and Y. Li, "Adaptive fuzzy decentralized control for stochastic large-scale nonlinear systems with unknown dead-zone and unmodeled dynamics," Neurocomputing, vol. 135, pp. 367-377, 2014.

[30] Y.-H. Chang and W.-S. Chan, "Adaptive dynamic surface control for uncertain nonlinear systems with interval type-2 fuzzy neural networks," IEEE Transactions on Cybernetics, vol. 44, no. 2, pp. 293-304, 2014.

[31] G.-O. Tirian, I. Filip, and G. Proştean, "Adaptive control system for continuous steel casting based on neural networks and fuzzy logic," Neurocomputing, vol. 125, pp. 236-245, 2014.

[32] H. Wang, P. X. Liu, and B. Niu, "Robust fuzzy adaptive tracking control for nonaffine stochastic nonlinear switching systems," IEEE Transactions on Cybernetics, vol. 48, no. 8, pp. 2462-2471, 2018.

[33] H. Wang, X. Liu, B. Chen, and Q. Zhou, "Adaptive fuzzy decentralized control for a class of pure-feedback large-scale nonlinear systems," Nonlinear Dynamics, vol. 75, no. 3, pp. 449-460, 2014.

[34] H. Shen, F. Li, H. Yan, H. R. Karimi, and H.-K. Lam, "Finitetime event-triggered $H_{\infty}$ control for T-S fuzzy Markov jump systems," IEEE Transactions on Fuzzy Systems, vol. 26, no. 5, pp. 3122-3135, 2018.

[35] X.-H. Chang and G.-H. Yang, "Nonfragile $H_{\infty}$ filter design for T-S fuzzy systems in standard form," IEEE Transactions on Industrial Electronics, vol. 61, no. 7, pp. 3448-3458, 2014.

[36] X.-H. Chang, J. Xiong, and J. H. Park, "Fuzzy robust dynamic output feedback control of nonlinear systems with linear fractional parametric uncertainties," Applied Mathematics and Computation, vol. 291, pp. 213-225, 2016. 